\title{
Animación hotelera. Una perspectiva para diferenciar la marca. Caso Club Amigo, grupo Cubanacán.
}

\section{Hoteliers Animation. A perspective to differenciate the brand. Club Anigo case, Cubanacan group.}

\author{
MSc. Yudiht Sánchez Romero ${ }^{1} \&$ DrC. Zoila Ulacia Oviedo ${ }^{2}$
}

\section{Resumen.}

El presente trabajo tiene como objetivo, rediseñar la conceptualización de la marca Club Amigo del grupo hotelero Cubanacán, desde la perspectiva de la animación, tomando en consideración el fenómeno marcario, posicionar un producto-servicio, es aquello que nos diferencia de nuestra competencia y no es más que, nuestra fortaleza plasmada en beneficios y atributos que poseamos a nuestro favor. En este sentido, la aplicación de métodos y técnicas como: análisis y síntesis, inducción/deducción, histórico-lógico, revisión bibliográfica y documental, así como, la observación directa, la encuesta, la entrevista, el criterio de expertos, técnicas estadísticas-matemáticas como el muestreo, análisis de frecuencias, el coeficiente de experticia, tablas y gráficos, permitieron determinar los beneficios intangibles y atributos que favorecen el rediseño de dicha marca para ser identificada por los clientes que opten por ella y se diferencie de las otras marcas del grupo hotelero.

Palabras claves: animación hotelera, rediseño, marca Club Amigo, conceptualización.

\section{Abstract.}

The objetive of the actual work is to redisign the conceptualization of the Club Amigo brand of the hotelier group Cubanacan, from the perspective of the animation, taking into account the brand phenomena, somewhat looking for a position to a product-service that is what differenciate us to the competition and is no other that aur strengh expressed in benefits and atributes in our behalf, in this way the aplication of methods and syntexs, induction and deduction, historical-logical, bibliographic revision and cocumental, as well as the direct observation, the encuesta, the interview, thr experts criteria statistics techiques on a matematics such as the sampling, frecquence analysis, the espertisse coeficiency, charts and graphies, allowed to determine the intangible benefits and

\footnotetext{
${ }^{1}$ Universidad de la Habana, Facultad de Turismo. La Habana, Cuba, yudith.sanchez@ftur.uh.cu

${ }^{2}$ Universidad de la Habana, Facultad de Turismo. La Habana, Cuba, zoila_ulacia@ftur.uh.cu
} 
attributes that favour the redisign of this brand to be identified by the clients and it differenciates from the other brand of the hotels group.

Keywords: hoteliers animation, redisign, Club Amigo brand, conceptualization.

\section{Introducción}

En las últimas décadas, el turista ve en sus vacaciones una experiencia con múltiples aportaciones: reconocimiento e integración del medio ambiente que visita; encuentros con otras culturas; descubrimiento de la historia y la evolución de otros pueblos, y una mayor participación dentro del desarrollo de las actividades, quieren ser sorprendidos durante su estancia con nuevas experiencias y recuerdos para llevarse a casa.

La animación turística ha sido un elemento que puede propiciar la transformación de los productos que ofertan las empresas del sector.

Una de las problemáticas que se evidencian en la actividad hotelera cubana, es la no diferenciación en el mercado, de una gran parte de las marcas diseñadas, aun cuando el Concepto de estas, se haya establecido con anterioridad. Otro aspecto a destacar es la no correspondencia directa entre la marca y la actividad de animación en estos establecimientos; los programas de animación de la mayoría de las entidades hoteleras, ya sean de sol y playa, ciudad y/o naturaleza, están diseñados con contenidos muy parecidos, por lo que no reflejan los atributos que la marca sugiere, para aquellos que no la conocen, ni despiertan el interés, para los que la conocen.

El Grupo CUBANACAN S.A, creada el 5 de agosto de 1987, no escapa a la situación antes descrita. De sus cinco marcas, las dos concebidas para Sol y Playa (Brisas y Club Amigo), reflejan frente al mercado, prácticamente lo mismo, lo que deviene su elección por el cliente, a través de la categoría o al espacio turístico que por sus características se asocian, entre otras cuestiones, al régimen All Inclusive. Uno de los aspectos que las hacen similares es la actividad de animación, existiendo, sin embargo, potencialidades de poderlas diferenciar mediante la misma.

Aunque el tema a tratar aún no ha cobrado fuerza, este representa un aspecto a considerar seriamente, si se quiere contar con un producto diferenciador, en tanto, la esencia de la marca es distinguir el producto-servicio.

Es por ello que, el objetivo general de la investigación, se define de la siguiente manera:

$>$ Rediseñar la conceptualización de la marca Club Amigo, del Grupo Cubanacán, desde la perspectiva de la animación. .

Para el desarrollo de la investigación, se emplearon métodos teóricos, empíricos y estadístico-matemáticos, aplicando los respectivos instrumentos para cada uno de ellos.

Lo novedoso del trabajo radica en que hasta el momento, a pesar que se han realizado varios estudios e investigaciones sobre la animación, estos no han estado orientados a determinar los beneficios y los atributos que sugieren las marcas en el mercado, desde la perspectiva de la animación, para diferenciar las mismas. 
El rediseño propuesto de la conceptualización de la marca desde la perspectiva de la animación de los clientes, brinda beneficios vivenciales, sensoriales y simbólicos o de status que son lo que estos buscan esencialmente, junto al beneficio material, en su relación con el producto-servicio.

\section{Métodos}

La trayectoria metodológica de esta investigación se sustentó en cuatro grandes fases, las que mostraron además, las técnicas e instrumentos que se emplearon en cada una de ellas para la recogida y análisis de información, que a continuación se describen:

\section{Fase 1. Establecimiento del marco conceptual y metodológico de la investigación}

El objetivo de esta primera fase estuvo centrado en construir el marco teórico conceptual y metodológico sobre la base de las temáticas vinculadas al fenómeno objeto de estudio, sintetizando y emitiendo criterios y juicios en este sentido. Por ello, se utilizó el método teórico, empleándose el análisis-síntesis, la inducción-deducción, las cuales permitieron profundizar en el conocimiento de las principales características y regularidades de la actividad.

El método histórico-lógico, se utilizó para describir la evolución del fenómeno de la animación hasta los momentos actuales, partiendo de sus antecedentes, además de la revisión bibliográfica y documental, las que apoyaron la búsqueda de la información pertinente, lo cual quedó reflejado el presente informe de investigación.

Fase 2. Identificación de los aspectos relevantes que caracterizan la marca desde la perspectiva de la animación

El objetivo de esta fase permitió poder identificar cuáles eran los aspectos relevantes que caracterizan a la marca hotelera Club Amigo, desde la perspectiva de la animación, contando con los criterios de los clientes que visitan estos hoteles y de los animadores.

Las técnicas e instrumentos aplicados fueron: la encuesta, la entrevista y la observación participante, análisis de frecuencia y técnica de muestreo, apoyadas por las herramientas estadístico-matemáticas que correspondían en cada caso. Otra de las técnicas empleadas fue la revisión documental.

La encuesta tuvo como objetivo identificar cuáles son los principales atributos que para los clientes tiene la marca Club Amigo, desde la perspectiva de la animación, así como, poder identificar las preferencias respecto a este producto-servicio. Fue elaborada en función de dos variables, una que se refiere a los atributos de la marca y la otra, a las actividades propiamente de animación.

Para aplicar el instrumento a los clientes extranjeros, se escogieron tres (3) hoteles de los seis (6) Club Amigo que existen en el país, representando esta cifra el 50\%. La 
determinación de la población de clientes a encuestar, tomó en consideración dos criterios:

$\checkmark$ Que fueran huéspedes de los hoteles objetos de estudio.

$\checkmark$ Que fueran solo extranjeros, por el concepto de compra que tienen, partiendo de sus necesidades y los atributos que conocen a través de la cultura de marcas.

Estos criterios condujeron a determinar la población durante tres días de estancia (fin de semana) comprendida en los meses de febrero a marzo del año 2017, momento en que se realizó la recogida de datos, (27 días).

Del total de 680 clientes alojados el tamaño de la muestra fue calculado a través de la siguiente fórmula:

$$
\mathrm{n}=\frac{\mathrm{N} * Z^{2} * p * q}{e^{2} *(N-1)+Z^{2} * p * q}
$$

Para un $95 \%$ de confiabilidad, dio un tamaño de muestra de 246 clientes extranjeros a encuestar.

La entrevista se realizó con el objetivo de establecer el nivel de correspondencia entre los criterios dados por los clientes, sobre los atributos y preferencias asociados al objeto de estudio y el de los animadores, incluyendo a los jefes de recreación, considerando que debían estar en la plantilla de los hoteles, cuyo censo fue de doce en total. Entre los requisitos de selección, se estableció el tiempo de experiencia en la marca de dicho personal, por lo que condujo a entrevistar a nueve del total antes dicho.

La guía de entrevista se elaboró, buscando la misma información que en la encuesta, pero en este caso, se abordaron siete aspectos que de manera general, estaban distribuidos también en dos variables, una sobre la marca Club Amigo y sus atributos, (comprende de la pregunta 1 a la 4) y la segunda variable asociada a las actividades de animación (comprende de la pregunta 5 a la 7).

A los fines de validar la información obtenida e identificar las condiciones materiales, de infraestructura y de apoyo a la actividad de animación, fue utilizada la observación participante, estructurada a partir de los siguientes elementos.

$\checkmark$ Entorno.

$\checkmark$ Las actividades de animación dentro de la instalación,

- Si aparecen los atributos que para clientes y animadores caracterizan la marca.

- Qué tipo de actividades prefieren.

- $\quad$ El nivel de participación de los clientes en las actividades.

- $\quad$ Las áreas del hotel que se vinculan a la animación.

$\checkmark$ Desempeño del personal.

A partir de la apreciación del objeto de estudio en sus múltiples manifestaciones y en toda su complejidad, se agrupa la información recogida y se contrasta con los resultados de las 
encuestas y las entrevistas, estableciéndose luego, los puntos coincidentes y los que difieren.

La revisión documental tuvo como finalidad, examinar el proceso de planificación de la actividad de animación en cada uno de los hoteles (Proyecto Operativo de Animación), en busca de la existencia de un enfoque sistémico y una gestión integrada de la misma. Así mismo, se indagó sobre la conceptualización actual de la animación en la marca Club Amigo a través de la revisión del Manual operativo de animación.

Fase 3. Determinación de los principales atributos que evoca la marca desde la actividad de animación

Se considera esta fase importante, en tanto completa toda la información necesaria que conduce al propósito de la investigación, facilitando el camino hacia la última fase. Su objetivo es, determinar los principales atributos que evoca la marca objeto de estudio en el mercado, desde la actividad de animación.

El punto de partida fue el análisis del Concepto actual de la marca, se listan los atributos identificados y se enriquecen con los resultados obtenidos de la variable uno de las encuestas a clientes y entrevista a animadores. Este proceder da paso a un listado preliminar de atributos, al que le fue necesario agregar el criterio, en relación a la cuestión abordada, de los directivos de cada uno de los hoteles estudiados. A tales efectos, se elaboró una encuesta para los directivos, donde solo se les facilitó el listado de atributos, con el propósito de obtener un listado definitivo, para luego legitimar toda la información recopilada sobre los atributos con criterios de expertos.

Para la aplicación de esta técnica, se tomaron a 16 posibles candidatos a expertos. Los criterios de selección fueron: tener conocimientos sobre animación hotelera; tener conocimientos generales sobre marcas hoteleras; contar como mínimo con 10 años dentro del sector turístico, (en hotel o escuela) en la especialidad de animación; haber dirigido la actividad de animación dentro del ámbito hotelero, a diferentes niveles por 3 años o más; tener de 3 a 5 años de experiencia en la animación de los hoteles marca Club Amigo, con conocimiento del perfil de los mercados que operan.

A estos 16 candidatos se les aplicaron los instrumentos asociados al Coeficiente de Experticia (k), a los fines de valorar su nivel de conocimiento sobre el tema (kc) y la fuente de argumentación del mismo en los criterios del experto (ka).

Luego de este análisis, se utilizaron para la consulta aquellos expertos que tuvieron un nivel de competencia alto, mediante los resultados de coeficientes de conocimiento (Kc), de argumentación y fundamentación (Ka) y de competencia $(\mathrm{K}=\mathrm{Kc}+\mathrm{Ka} / 2)$. Por lo que resultaron expertos, un total de 10 declarados.

\section{Fase 4. Conceptualización de la marca desde la actividad de animación}

Esta última fase de la trayectoria metodológica, tiene como objetivo definir los elementos que conforman la conceptualización de la marca Club Amigo, desde la actividad de animación. En la misma, se partió del análisis de los resultados de los instrumentos 
aplicados en las fases anteriores, para arribar a la propuesta que se persigue se tomó en consideración los resultados obtenidos en la entrevista aplicada a los Expertos, atendiendo a tres aspectos a abordar: lo que la marca Club Amigo le sugiere al mercado; los atributos percibidos de la marca; los atributos que deberían desarrollarse para fortalecer y consolidar la marca en el mercado.

\section{Resultados}

Respecto a lo que significa la marca para los clientes, el resultado se muestra en la siguiente figura:

Figura 1: Significado de la marca para los clientes

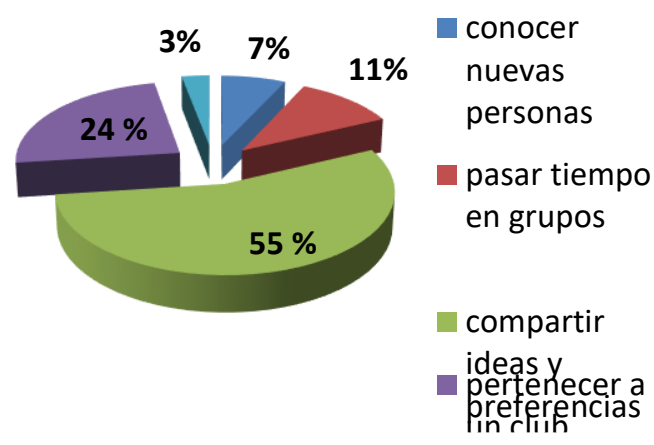

Fuente: Elaboración propia

En lo referido a los elementos que caracterizan mayormente los hoteles de la marca "Club Amigo", desde la perspectiva de los clientes, permitió identificar diez elementos prioritarios.

Tabla 1. Prioridad y evaluación de los elementos que caracterizan la marca Club Amigo desde la perspectiva de los clientes

\begin{tabular}{|l|c|c|c|}
\hline \multicolumn{1}{|c|}{ ELEMENTOS SELECCIONADOS } & $\%$ & $\begin{array}{c}\text { ORDEN DE } \\
\text { IMPORTANCIA }\end{array}$ & EVALUACIÓN \\
\hline Familiaridad & 80 & 2 & Muy Bien \\
\hline Alegría & 35,7 & 5 & Muy Bien \\
\hline Diversión & 38,6 & 4 & Bien \\
\hline Hacer las cosas junto a otras personas & 41,8 & 3 & Regular \\
\hline Mucha actividad & 23,1 & 7 & Regular \\
\hline Hoteles de sol y playa & 21,5 & 8 & Muy Bien \\
\hline Personal fundamentalmente joven, amable y alegre & 20,3 & 9 & Bien \\
\hline Servicios tematizados & 83,3 & 1 & Regular \\
\hline Ambiente relajante & 24,7 & 6 & Bien \\
\hline Otros & 8,5 & 10 & \\
\hline
\end{tabular}

Fuente: Elaboración propia 
De ellos más del $79 \%$ consideran como elementos de mayor importancia los servicios tematizados, familiaridad y hacer cosas junto, sumado a la alegría y la diversión, constituyó los cinco (5) atributos más significativos para los clientes, expresado en la tabla anterior.

En relación a la preferencia de actividades, la figura 2 refleja el criterio de los clientes en este sentido.

Figura 2: Preferencia de actividades por los clientes

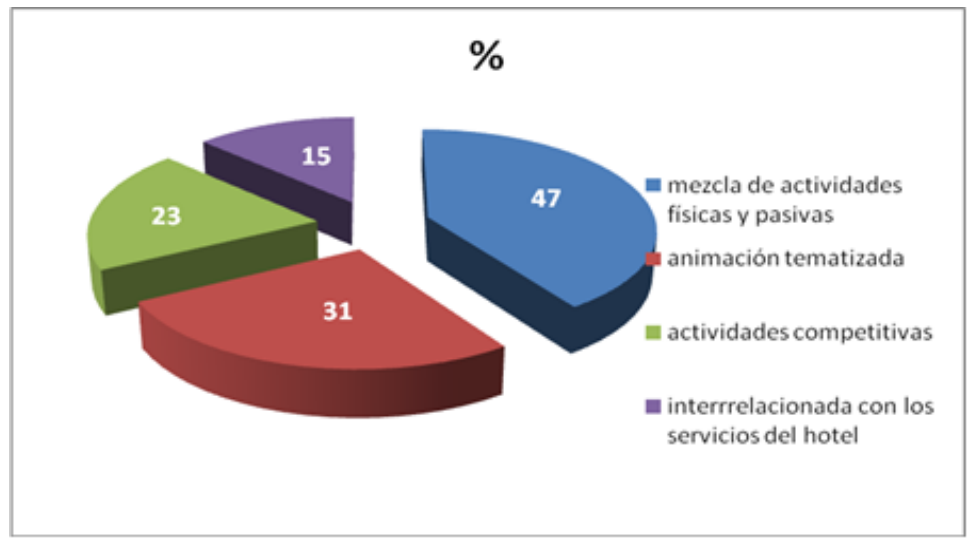

Fuente: Elaboración propia

El hecho de que en las preferencias de los clientes aparezcan en los dos primeros lugares, la mezcla de actividades físicas y pasivas y la animación tematizada, deduce en la combinación de tematizar la animación basada en actividades que mezclen estas dos variables.

Del tipo de actividades seleccionadas, los componentes de preferencia que señalaron los clientes son:

Figura 3: Componentes de las actividades tematizadas

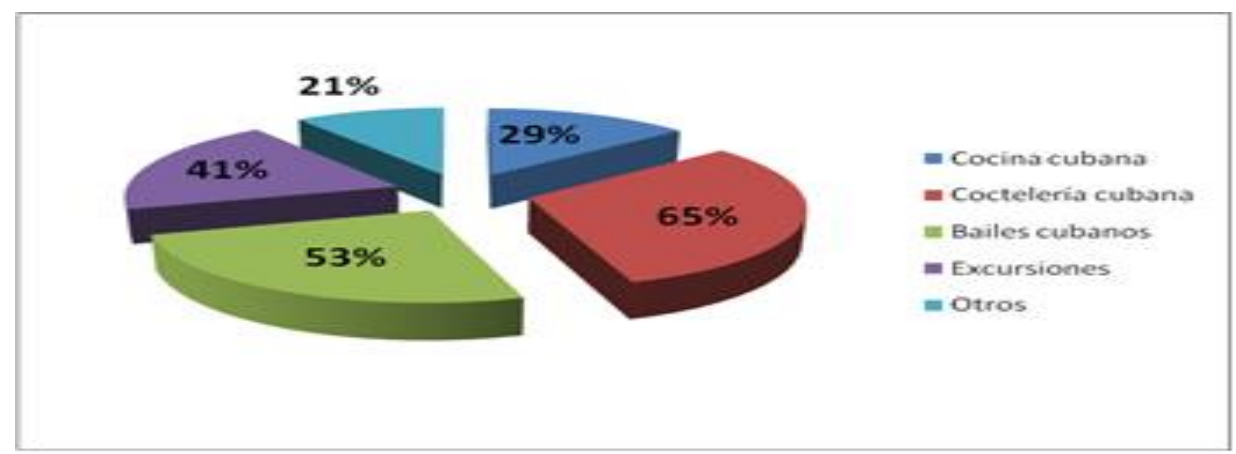

Fuente: Elaboración propia

> En relación a los servicios asociados a la animación, resultan pocos los encuestados que piensan en una asociación en este sentido, sin embargo, el análisis de las respuestas dadas por este grupo, evidenció que coincidieron el 72,36\% de los encuestados en asociar esta actividad a los bares y un $41,46 \%$ a los restaurantes y solo el 27, 23\% pensó en asociarla a la cocina. 
Lo más representativo sobre el resultado de la entrevista recogida del criterio de los nueve animadores en cuanto a los atributos refieren: la alegría, cubanía, amistad, familiaridad, diversión y animación asociada a diversos temas.

Ello conduce a concluir que no existe una diferenciación de los beneficios que sugiere la marca en cuestión, por ejemplo el elemento de cubanía se encuentra en una marca u otra y se aprecia que este personal no sabe identificar claramente que son beneficios y que son atributos, aunque los dos estén muy relacionados.

Los resultados de la observación directa teniendo en cuenta tres aspectos abordados en la guía permiten concluir que existen atractivos propicios para desarrollar una animación donde se vincule las potencialidades del entorno con el hotel, aprovechando todos los espacios lo que pudiera tenerse en cuenta en la conceptualización.

En cuanto al análisis documental se pudo acceder a los programas, guiones y fichas técnicas de las actividades, si bien dentro del Proyecto Operativo de Animación (POA), existe un punto referente a estos documentos rectores de la actividad, no existe el diseño del POA, lo que quiere decir que al no tenerlo elaborado y puesto en funcionamiento, denota que la planificación no tiene un enfoque coherente, integrador y sistémico, lo cual conlleva que los programas de animación se elaboran sin contar con el respaldo del conocimiento de las potencialidades del entorno (natural, social, cultural); así como, el estudio de los mercados y sus segmentos, es limitado. El análisis del Concepto corroboró la falta de coherencia entre lo que está normado y lo que se hace en la realidad, no denota una clara visión de los beneficios que se quieren brindar con la marca, sustentada por la recreación y la animación en general, es decir, que ella abarca todos los espacios atendiendo a una "filosofía de club", cuestión tal que en la práctica no se ejecutan programas que respalden dicha filosofía y por tanto no es percibida por los clientes.

El resultados de la encuesta a los directivos de las instalaciones objeto de estudio, trae consigo la lista de los atributos que a sus criterios sugiere dicha marca, a su vez, se muestran a continuación, los atributos que aparecen en el Concepto, los que sugieren los clientes y los animadores, los cuales no difieren y coinciden con el criterio de los directivos, expresado en este listado:

\begin{tabular}{|l|l|l|l|}
\hline $\begin{array}{l}\text { Atributos del concepto } \\
\text { actual }\end{array}$ & $\begin{array}{l}\text { Atributos } \\
\text { (Clientes) }\end{array}$ & $\begin{array}{l}\text { Atributos } \\
\text { (Animadores) }\end{array}$ & $\begin{array}{l}\text { Atributos } \\
\text { (Directivos) }\end{array}$ \\
\hline Ambientes sanos & $\begin{array}{l}\text { Servicios } \\
\text { tematizados }\end{array}$ & Alegría & Amistad \\
\hline Diversión & Familiaridad & Intercambio cultural & Familiaridad \\
\hline Alegria & Hacer cosas juntos & Amistad & Compartir con otros \\
\hline Mucha actividad & diversión & Familiaridad & Alegría \\
\hline & Alegría & Diversión & Diversión \\
\hline
\end{tabular}

Todo ello conlleva a listar los principales atributos que evoca la marca objeto de estudio, en el mercado, desde la actividad de animación como resultado de la tercera fase: servicios tematizados, diversión, alegría, compartir con otros, familiaridad, mucha actividad, clubes temáticos y confianza. 
A partir de los razonamientos anteriores unido al criterio de expertos se valida el listado de atributos que, como se puede apreciar los mismos coinciden hacia: la familiaridad, la diversión, la alegría, los servicios tematizados, la formación de clubes por segmentos de mercados, gustos y preferencias. Todo ello, a los fines de tomarlos en consideración para conceptualizar la marca desde la animación, se toma como referencia de las experiencias anteriores de conceptualizaciones de la animación en las marcas del grupo hotelero objeto de estudio, ocho componentes para la propuesta del rediseño, los cuales se exponen a continuación.

Figura 4 Componentes de la conceptualización de la marca Club Amigo.

\begin{tabular}{|c|c|}
\hline $\begin{array}{l}\text { ORIENTACIÓN } \\
\text { AL MERCADO }\end{array}$ & $\begin{array}{l}\text { Las instalaciones Club Amigo están dirigidas a operar el } \\
\text { segmento familia. }\end{array}$ \\
\hline CONCEPTO & $\begin{array}{l}\text { Está sustentado en la ANIMACIÓN } \text { EN TODOS LOS } \\
\text { ESPACIOS, SOBRE LA BASE DE LA DIVERSIDAD } \\
\text { RECREATIVA QUE GENERA CONOCIMIENTO, } \\
\text { ALEGRÍA, AMISTAD Y CONFIANZA }\end{array}$ \\
\hline ATRIBUTOS & $\begin{array}{l}\text { - } \frac{\text { Servicios tematizados }}{\text { - }} \\
\text { - } \frac{\text { Varividades de carácter familiar opciones recreativas }}{\text { - Intercambio de saberes }} \\
\text { - } \\
\text { Diversión }\end{array}$ \\
\hline SLOGAN & "En la diversión y en todos los espacios, AMIGOS" \\
\hline LOGOTIPO & $\begin{array}{l}\text { Puede ser igual al ya existente, pero predominando el color } \\
\text { naranja, (se logra con la mezcla del color rojo y el amarillo). }\end{array}$ \\
\hline & $\begin{array}{l}\text { La estructura operativa descansa en la conformación de clubes } \\
\text { atendiendo a los gustos y preferencias manifestados por los } \\
\text { clientes y generados, además, por lo que sugiere la marca. Estos } \\
\text { clubes son: }\end{array}$ \\
\hline ESTRUCTURA & $\begin{array}{l}\text { - Club de cocina "Fogón a la vista" } \\
\text { - Club de Coctelería "Constantino's Bar" } \\
\text { - Club de baile "Bailar, sentir, vivir" } \\
\text { - Club de Naturaleza "Descubre el entorno" } \\
\text { - Club Infantil "Ciudad de números y letras" }\end{array}$ \\
\hline $\begin{array}{l}\text { EQUIPO DE } \\
\text { ANIMACIÓN }\end{array}$ & $\begin{array}{l}\text { Conformado por un jefe de recreación y } 6 \text { a } 8 \text { animadores cuyas } \\
\text { características, además de los requisitos técnicos, tengan } \\
\text { especialización en deportes, animación infantil, conocimientos } \\
\text { de restauración, sepan bailar bien, dominio de } 2 \text { idiomas y } \\
\text { nociones de otro (en dependencia de los mercados que operan } \\
\text { estos hoteles). El rango de edad, oscila entre } 20 \text { - } 30 \text { años. Uso de } \\
\text { uniforme reglamentario, de acuerdo al diseño de la marca para el } \\
\text { día y para la noche de modo que se identifiquen fácilmente. }\end{array}$ \\
\hline
\end{tabular}

Fuente: Elaboración propia 


\section{Discusión}

1. Orientación al mercado: Respecto a la orientación al segmento de familia se considera mantenerlo, respetando la fundamentación de los estudios realizados por el corporativo en cuanto al mercado.

2. Concepto: Sobre la base del Concepto actual que plantea: "ANIMACIÓN EN TODOS LOS ESPACIOS”, diseñándose diferentes opciones temáticas en correspondencia con las características particulares de cada instalación”, y con el análisis realizado a dicho Concepto, se toman aspectos que resultan válidos para la propuesta de este trabajo, enriqueciendo el mismo a partir de los resultados obtenidos antes expuestos, debido a ello se enfatizan beneficios intangibles tales como la diversidad recreativa, el conocimiento, la alegría, la amistad y la confianza.

3. Atributos: Como se puede observar los atributos propuestos guardan una relación muy estrecha con los beneficios intangibles formulados en el Concepto, lo que pudiera producir algún tipo de confusión. Los beneficios se manifiestan a través de atributos, es decir, un beneficio puede convertirse en una característica del producto y son expresados como atributos, tal es el caso por ejemplo, del atributo servicios tematizados cuya expresión como beneficio son la diversidad y el conocimiento.

4. Slogan: No es una camisa de fuerza, la propuesta no difiere en gran medida del que ya existe.

5. Logotipo: Se propone el predominio del color naranja que según estudios realizados sobre psicología del color, evoca conocimiento y creatividad, dos de los aspectos claves que genera la animación; por otra parte, se ha tomado como referencia del llamado "turismo naranja" (Tresserras, 2015) que promociona, entre otros beneficios, una oferta turística con la segmentación de productos/servicios eficientes e innovadores.

6. Estructura (Clubes): Para su determinación se tomaron los análisis realizados de los resultados por la aplicación de los métodos, técnicas y herramientas que sustentan a la investigación.

7. Equipo de animación: La cantidad de animadores que conforman el equipo de animación, está en dependencia no solamente de la temporada, sino también lo que en este sentido se maneja en los hoteles del Caribe y Centro América sobre la contratación del personal vinculado a la actividad, este número de integrantes, está por debajo de la cantidad de animadores que operan la recreación y la animación en las cadenas hoteleras de estas regiones. Se sugiere que el equipo de animación esté vinculado estrechamente al talento artístico contratado en el hotel y por supuesto, todo ello debe estar en correspondencia a los estándares establecidos por la marca.

8. Caracterización de espacios. Estándares: Están asociado a los contenidos a desarrollar por cada club y los estándares que lo caracterizarán, estos se exponen a continuación: 
Tabla 2. Clubes y estándares asociados a los atributos de la marca Club Amigo

\begin{tabular}{|c|c|c|}
\hline CLUB & CONTENIDO & ESTANDARES \\
\hline $\begin{array}{c}\text { "Fogón a la } \\
\text { vista" }\end{array}$ & $\begin{array}{l}\text { Orientado a clientes que gustan conocer } \\
\text { sobre la cocina cubana y su elaboración. } \\
\text { Las actividades del mismo, están } \\
\text { concebidas sobre la base de talleres, } \\
\text { exposiciones, demostraciones y } \\
\text { competencias. Los espacios asociados al } \\
\text { club son los restaurantes, piscina y la } \\
\text { cocina. Algunas de estas actividades } \\
\text { pueden ser por ejemplo: } \\
\text { Taller: "Menú a la carta" } \\
\text { Competencia: "El jugo favorito" }\end{array}$ & $\begin{array}{l}\text { Las recetas de cocina } \\
\text { que se seleccionen } \\
\text { deben ser sencillas y } \\
\text { rápidas de hacer. } \\
\text { Recetas de la cocina } \\
\text { tradicional cubana. } \\
\text { Inclusión de recetas } \\
\text { característica de la } \\
\text { región donde esté } \\
\text { enclavado el hotel. }\end{array}$ \\
\hline $\begin{array}{c}\text { "Constantino's } \\
\text { Bar" }\end{array}$ & $\begin{array}{l}\text { Orientado a clientes que desean conocer } \\
\text { aspectos de la coctelería cubana y } \\
\text { aprender a elaborar algunos de los } \\
\text { cocteles más conocidos. Es un club } \\
\text { dedicado a uno de los más reconocidos } \\
\text { Bar ténder cubano. Los espacios que } \\
\text { contemplan este club están referidos a los } \\
\text { bares del hotel, los ranchones de la playa. } \\
\text { Las actividades que conforman el club } \\
\text { están basadas en talleres, competencias, } \\
\text { degustaciones y demostraciones, como } \\
\text { por ejemplo, } \\
\text { Competencia: “Compite y gana" } \\
\text { Demostración: "El arte del Bartender" }\end{array}$ & $\begin{array}{c}\text { La existencia de un } \\
\text { coctel que identifica al } \\
\text { club. } \\
\text { Privilegiar bebidas } \\
\text { analcohólicas. }\end{array}$ \\
\hline $\begin{array}{c}\text { "Bailar, sentir, } \\
\text { vivir" }\end{array}$ & $\begin{array}{l}\text { Orientado a clientes que gusten de la } \\
\text { música y el baile. Pueden ser miembros } \\
\text { del club aquellos grupos danzarios y } \\
\text { escuelas de baile originarios de otros } \\
\text { países. Los espacios para el desarrollo de } \\
\text { las actividades están contemplados en el } \\
\text { área de playa, la piscina, el área de } \\
\text { espectáculos y las salas de fiesta, de } \\
\text { contarse con ella. Se propone actividades } \\
\text { de carácter competitivo, talleres, } \\
\text { demostraciones y juegos. Ej. } \\
\text { Taller: “Un dos tres, un dos” } \\
\text { Demostración: “Tarde de la Rumba” }\end{array}$ & $\begin{array}{c}\text { Tematizar las } \\
\text { actividades. } \\
\text { Géneros músico- } \\
\text { danzarios cubanos y } \\
\text { caribeños. }\end{array}$ \\
\hline
\end{tabular}




\begin{tabular}{|c|c|c|}
\hline $\begin{array}{c}\text { "Descubre el } \\
\text { entorno" }\end{array}$ & $\begin{array}{l}\text { Orientado a clientes amantes de la } \\
\text { naturaleza y el descubrimiento. Los } \\
\text { espacios asociados son el entorno natural } \\
\text { del hotel en cuestión. Las actividades } \\
\text { fundamentales son: Excursiones, Talleres } \\
\text { y Exposiciones. Ejemplo de ellas son: } \\
\text { Excursión: "Por senderos, a caballo" } \\
\text { Taller: “Confeccione su trofeo" }\end{array}$ & $\begin{array}{c}\text { Animadores jóvenes, } \\
\text { especializados en el } \\
\text { tema. } \\
\text { Trofeos de origen } \\
\text { natural. }\end{array}$ \\
\hline $\begin{array}{c}\text { "Ciudad de } \\
\text { números y } \\
\text { letras" }\end{array}$ & $\begin{array}{l}\text { Orientado al segmento infantil. Se } \\
\text { concibe como espacio para este club, la } \\
\text { creación de una pequeña ciudad al aire } \\
\text { libre, con un área pequeña de descanso y } \\
\text { estancia, dividida por edades. Contará } \\
\text { con parque de diversiones sencillo, un } \\
\text { área de juegos tradicionales cubanos, } \\
\text { equipos de juegos electrónicos. El diseño } \\
\text { constructivo y espacial de las áreas se } \\
\text { basará en números y letras. }\end{array}$ & $\begin{array}{c}\text { Animadores } \\
\text { especializados y los } \\
\text { más jóvenes. } \\
\text { Uso de materiales e } \\
\text { insumos inocuos para } \\
\text { los infantes. }\end{array}$ \\
\hline
\end{tabular}

Fuente: Elaboración propia

\section{Conclusiones}

$>$ Las técnicas empleadas durante el desarrollo de la investigación, en particular la retroalimentación de los clientes permitieron formular el Concepto de la marca desde la perspectiva de la animación, lo que permite la gestión de esta con mayor orientación hacia el cliente, en consecuencia garantiza la satisfacción del mismo.

> Los atributos de la marca Club Amigo que resultan más importantes para los clientes fueron: servicios tematizados, familiaridad, hacer cosas juntos, lo que implicó beneficios intangibles como la diversidad, el conocimiento, la amistad y la confianza.

La propuesta refleja que el diseño de actividades de animación, reflejado en la estructura por clubes, atendiendo al Concepto de la marca, permite una mejor identificación de esta última en el mercado.

\section{Referencias Bibliográficas}

De La Tejera, E. Manual Operativo de Animación Turística y Desarrollo Sustentable. Ediciones Balcón. FORMATUR. 2007.

De la Ribera Blanco, Luís E y Rodríguez Álvarez, Aloida L. La magia del color. CIATUR, 2000.

Grupo Cubanacán. Informes de calidad y desarrollo. Dirección de Calidad. Ministerio de Turismo de la República de Cuba. Años del 2013 al 2017. 
Grupo Hotelero Cubanacán. "Bases conceptuales de la marca Club Amigo". Manual Operativo de Explotación. Dirección de Explotación, Casa Matriz. 2016.

Grupo Hotelero Cubanacán. Manuales operativos de recreación y animación de las marcas Brisas y Club Amigo. 2016.

Hernández, Sampier: “ Metodología de la Investigación parte 1“.Editorial Félix Varela. 75. La Habana. 2004.

MINTUR-FORMATUR. Informe nacional sobre la evaluación del proceso de animación. Taller Nacional de Calidad. La Habana, 2017.

Sainz de Robles. Diccionario Español de Sinónimos y Antónimos. Edición Revolucionaria. Instituto Cubano del Libro, 1968.

Tresserras Juan J. El turismo naranja, el color del turismo cultural y creativo. Revista de Economía Creativa. Santiago Creativo $n^{\circ}$ 1: 51-52. Programa CORFO del Ministerio de Economía de Chile. Santiago. 2015. (Consultado: julio 2016). Disponible http//www.boletinturistico.com.

Sales Bressel, Paloma. Innovando la Animación Turística. Revista Hosteltur [en línea] Marzo/2018 (Consultado: Mayo 2018) Disponible http/www.hosteltur/comunidad.palomasales@fiestaconsulting.com.

Sitios en internet referido a: Teoría del color. (Consultado: febrero 2018) Disponible en http:// www.xtec.es/ aromero8/index.htm./todacultura.com

\section{LCiencia}




\section{Para citar el artículo indexado.}

Sánchez Y., \& Ulacia Z. (2018). Animación hotelera. Una perspectiva para diferenciar la marca. Caso Club Amigo, grupo Cubanacán. Revista electrónica Explorador Digital 2(4), 34-47. Recuperado desde:

http://cienciadigital.org/revistacienciadigital2/index.php/exploradordigital/article/view/339/7 $\underline{51}$

\section{Ciencia \\ Digital \\ Editorial}

El artículo que se publica es de exclusiva responsabilidad de los autores y no necesariamente reflejan el pensamiento de la Revista Explorador Digital.

El articulo queda en propiedad de la revista y, por tanto, su publicación parcial y/o total en otro medio tiene que ser autorizado por el director o editor de la Revista Explorador Digital.
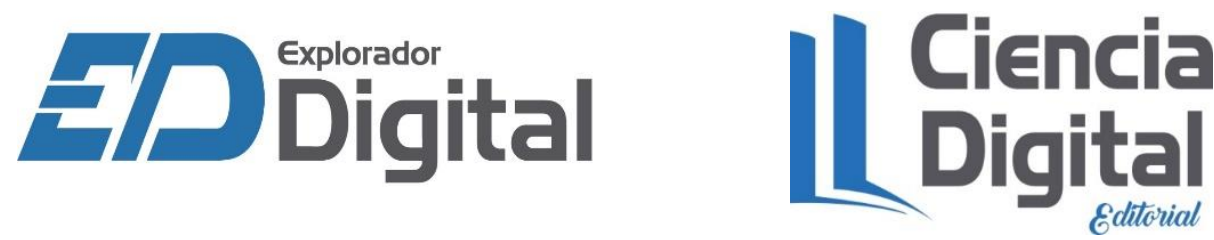